\title{
Risk-Sensitive Control for Sampled-Data Systems
}

\author{
Jun Yoneyama \\ Department of Electrical and Electronic Engineering \\ Faculty of Engineering, Shizuoka University \\ 5-1 Johoku 3-chome, Hamamatsu, Shizuoka 432 Japan
}

\begin{abstract}
The optimal stocahstic control of jump systems with sampled inputs and observations, which minimizes the expected value of an exponential cost criterion is considered. The change of measure technique is employed to obtain the information state for the problem, which is the sufficient statistics. The optimal controller which optimizes the exponential cost criterion is derived through a combination of both continuous-time and discrete-time Riccati equations. The various limits of the problem are also considered.
\end{abstract}

\section{Introduction}

There has recently been much attention to sampleddata systems. In practice, although the state of the system may be evoled through a continuous-time function, the observation of the system output is measured in discrete instants of time and thus the controller that is usually constructed through the sampled observation is also a discrete-time function. Such a sampled-data system is quite applicable to many practical models, and the theoretical design of sampled-data systems is required in many applications.

For the sampled-data systems, numerous works have been made and have appeared in the literature. In this paper, the optimal stochastic control problems with exponential cost criteria for the sampled-data systems are considered. The optimal controls of linear systems with exponential cost criteria have been obtained in [3], [5], [6]. In addition, the relation to game problems have been discussed. Motivated by Bensoussan's idea [1], [2], we use the information state to solve the problem. We obtain the explicit controller for LEQG problem. Finally, we analyze various limits of the problem.

\section{Risk-sensitive Optimal Con- trol}

\subsection{Problem Formulation}

On a probability space $(\Omega, \mathcal{A}, P)$ we consider a jump system described by

$$
\begin{aligned}
& d x=f(x, t) d t+\sqrt{\varepsilon} g(x, t) d w, t \neq k h, \\
& x\left(k h^{+}\right)=f_{d}\left(x, u_{k}, k h\right)+\sqrt{\varepsilon} g_{d}(x, k h) w_{k}, \\
& x(0)=x_{0}+\sqrt{\varepsilon} \hat{x}_{0} . \\
& y_{k+1}=h(x, k h)+\sqrt{\varepsilon} v_{k},
\end{aligned}
$$

where $x(\cdot) \in \Re^{n}$ is the state with jumps in each sample time; $w(\cdot) \in \Re^{p}$ is the process noise; $u_{k} \in \Re^{m}$ is the control; $w_{k} \in \Re^{p}$ is the process noise; $y_{k} \in \Re^{r}$ is the observed output; $v_{k} \in \Re^{r}$ is the measurement noise. $\varepsilon$ is a positive scalar called a small-noise parameter. We assume the followings.

Assumption 2.1 1) $x(0)$ is a random variable with gaussian probability law with mean $x_{0}$ and covariance matrix $\varepsilon P_{0}$.

2) $w(\cdot)$ is a Wiener process with covariance matrix $W(\cdot)$.

3) $w_{k}$ is a white Gaussian sequence with covariance matrix $W_{k}$.

4) $y_{0}=0$.

5) $v_{k}$ is also a white Gaussian sequencce with covariance matrix $V_{k}>0 \forall k$.

6) The process $w(\cdot)$, the sequences $w_{k}$ and $v_{k}$ and the variable $x(0)$ are mutually independent.

7) $f \in C^{1}\left(\Re^{n}, \Re^{n}\right)$ is bounded and uniformly continuous.

8) $g \in C\left(\Re^{n}, \Re^{n}\right)$ is bounded and uniformly continuous.

9) $f_{d} \in C^{1}\left(\Re^{n} \times \Re^{m}, \Re^{n}\right)$ is bounded and uniformly continuous.

10) $g_{d} \in C\left(\Re^{n}, \Re^{n}\right)$ is bounded and uniformly continuous.

11) $h \in C\left(\Re^{n}, \Re^{r}\right)$ is bounded and uniformly continuous.

Now we define the cost criterion;

$$
\begin{aligned}
J(u)= & E \exp \left[\frac { \theta } { \varepsilon } \left\{\Phi\left(x, t_{f}\right)+\int_{0}^{t_{f}} L(x, t) d t\right.\right. \\
& \left.\left.+\sum_{k=0}^{N-1} M(u, k)\right\}\right]
\end{aligned}
$$

where $(N-1) h<t_{f}<N h$. Then the risk-sensitive optimal control problem is to find $u_{k}^{*}$ such that $J(u)$ defined by (5) is minimized;

$$
J\left(u^{*}\right) \leq J(u)
$$

where $u$ is restricted to a causal function of the measurement history $\mathcal{Y}^{k}$ defined by 
$\mathcal{Y}^{k}=\sigma\left(y_{l}, l \leq k\right)$.

We write the partial derivatives of the function $f(x, t)$ as follows;

$D_{x} f(x, t)=\frac{\partial f}{\partial x}, D_{x}^{2} f(x, t)=\frac{\partial^{2} f}{\partial x^{2}}$.

Similar notation will be used hereafter.

\subsection{Information State}

Following Bensoussan et al. in [1], [2], we can convert the original problem into an equivalent one with full state information.

In the partial state information system, the state is not directly observable. In this case, we would look for another property, namely, that the state an time $t$ be computable from the information available at time $t$, which is the measurement history $\mathcal{Y}^{k}$ and the control history

$\mathcal{U}^{k}=\sigma\left(u_{l}, l \leq k\right)$.

Kumar and Varaiya showed in [4] that the conditional probability density function of the cost criterion given the measurement history $\mathcal{Y}^{k}$, called an information state, does not depend on a control law and it can be evaluated from $\mathcal{Y}^{k}$, and $\mathcal{U}^{k}$. Thanks to this property, we use the information state to analyze the problem.

The use of Girsanov's theorem allows us to get much simpler form of the conditional expectation of the cost criterion given the measurement history, from which the information state is readily derived. In order to use Girsanov's theorem, we define the change of probability measure by

$$
\left.\frac{d P}{d \tilde{P}}\right|_{\mathcal{F}^{t}}=\eta_{k}=\Pi_{l=1}^{k} \psi\left(x((l-1) h), y_{l}\right)
$$

where

$$
\psi(x ; y)=\exp \left[\frac{1}{\varepsilon}\left[h^{T}(x) V^{-1} y-\frac{1}{2} h^{T}(x) V^{-1} h(x)\right]\right]
$$

and $\mathcal{F}^{t}$ denotes the complete filtration generated by

$$
\mathcal{F}^{t}=\sigma\left(x(\tau), \tau \leq t, y_{l}, l \leq k\right) .
$$

By Girsanov's theorem under this change of probability measure, $y_{k}$ is a white Gaussian sequence with the covariance matrix $\varepsilon V$ in the probability space $(\Omega, \mathcal{A}, \tilde{P})$. Moreover, $x(0), w, y$ remain mutually independent, $x(0), w$ keeping the same probability laws. Therefore, we convert the system (1)-(4) for a convenient probability space $(\Omega, \mathcal{A}, \tilde{P})$.

We calculate the conditional expectation of the cost criterion given the measurement history. Consider the cost criterion

$$
\begin{gathered}
J=E\left[\operatorname { e x p } \left\{\frac { \theta } { ( } \left(\Phi\left(x\left(t_{f}\right)\right)+\int_{0}^{t_{f}} L\left(x_{t}\right) d t\right.\right.\right. \\
\left.\left.\left.+\sum_{l=1}^{N-1} M\left(u_{l}\right)\right)\right\}\right] .
\end{gathered}
$$

We first consider the cost criterion for $k h^{+} \leq t \leq(k+$ 1) $h$. On these intervals, no input and output are made. Define

$$
N_{t}=\exp \left(\frac{\theta}{\varepsilon} \int_{k h^{+}}^{t} L_{1}\left(x_{t}\right) d \tau\right) .
$$

Then $M_{t}$ satisfies

$$
d N_{t}=\frac{\theta}{\varepsilon} N_{t} d t, N_{k h^{+}}=1 .
$$

For any function $\phi$, we write

$$
\sigma(\phi)_{t}=E\left[N_{t} \phi(x)\right] .
$$

In case the measure defined by $\sigma(\phi)_{t}$ has a density $q(x, t)$, we have

$$
\sigma(\phi)_{t} \triangleq<\phi, q>=\int_{\Re^{n}} \phi(x) q(x, t) d x .
$$

Now we obtain the cost criterion.

Lemma 2.1 Suppose that $\phi$ is a $C^{2}$ function. Then $\sigma(\phi)_{t}=\sigma(\phi)_{k h^{+}}+\frac{\theta}{\varepsilon} \int_{k h^{+}}^{t} \sigma(L \phi) d \tau+\int_{k h^{+}}^{t} \sigma(T \phi) d \tau($

where $T$ is the operator defined by

$$
T=f(x, t) \frac{\partial}{\partial x}+\frac{\varepsilon}{2} \operatorname{trg}(x, t) W g^{T}(x, t) \frac{\partial^{2}}{\partial x^{2}}
$$

and

$$
\sigma(\phi)_{0}=E\left[\phi\left(x_{0}\right)\right]=\varepsilon \int_{\Re^{n}} \phi(x) P_{0} d x .
$$

Proof: See [2].

The probability density function of the cost criterion is readily derived from Lemma 2. 1 .

Theorem 2.1 If $\phi(\cdot)_{t}$ has a density $q(x, t)$, then it satisfies

$$
\begin{aligned}
d q= & {\left[-D_{x} q(x, t) f(x, t)-q(x, t) \operatorname{tr} D_{x} f(x, t)\right.} \\
& +\frac{\theta}{\varepsilon} q(x, t) L(x, t) \\
& \left.+\frac{\varepsilon}{2} \operatorname{trg}(x, t) W g^{T}(x, t) D_{x}^{2} q(x, t)\right] d t, \\
q(x, 0)= & \frac{\exp \left\{-\frac{1}{2 \varepsilon}(x-\hat{x}(0))^{T} P_{0}^{-1}(x-\hat{x}(0))\right\}}{(2 \pi)^{n / 2}\left|\varepsilon P_{0}\right|^{1 / 2}} .
\end{aligned}
$$

We call $q(x, t)$ the information state.

Proof: See [2].

Remark 2.1 There exists a backward adjoint process $p(x, t)$ such that

$<p\left(x, t_{1}\right), q\left(x, t_{1}\right)>=<p\left(x, t_{2}\right), q\left(x, t_{2}\right)>$

for all $t_{1}, t_{2} \in\left[0, t_{f}\right]$ except $t_{1}, t_{2}=k h$. Actually, the process $p(x, t)$ satisfies the parabolic equation adjoint to (21) and (22), namely,

$$
\begin{aligned}
& -d p=\left[D_{x} p(x, t) f(x, t)+\frac{\theta}{\varepsilon} p(x, t) L(x, t)\right. \\
& \left.+\frac{\epsilon}{2} \operatorname{trg}(x) W g^{T}(x) D_{x}^{2} p(x, t)\right] d t, \\
& p\left(x, t_{f}\right)=\exp \left(\frac{\theta}{\varepsilon} \Phi\left(x, t_{f}\right)\right) .
\end{aligned}
$$

Next we consider the cost criterion at time $t=k h$. We define an information state $q(x, k h)$ by

$$
\begin{aligned}
& <q\left(x, k h^{+}\right), \phi(x)> \\
& \quad=\tilde{E}\left[\eta_{k+1} \exp \left(\frac{\theta}{\varepsilon} \sum_{l=0}^{k} M\left(u_{l}\right)\right) \phi(x) \mid \mathcal{Y}^{k+1}\right],
\end{aligned}
$$


and define the bounded operator $\Lambda(u, y)$ by

$$
\begin{aligned}
& \Lambda(u, y) p(x)=\int_{\Re^{n}} \frac{\exp \left(-\frac{1}{2 \varepsilon} w^{T} W^{-1} w\right)}{(2 \pi)^{p / 2}|\varepsilon W|^{1 / 2}} \\
& \quad \times p\left(f_{d}(x, u)+g_{d}(x) w\right) d w \exp \left(\frac{\theta}{\varepsilon} M(u)\right) \psi(x, y) .
\end{aligned}
$$

The following theorem shows that $q(x, k h)$ satisfies the recursive equation.

Theorem 2. 2 The information state $q(x, k h)$ satisfies the recursion

$$
q\left(x, k h^{+}\right)=\Lambda^{*}\left(u_{k}, y_{k+1}\right) q(x, k h)
$$

where $\Lambda^{*}(u, y)$ is adjoint to the operator $\Lambda(u, y)$, defined by

$$
<\Lambda(u, y) p, q>=<p, \Lambda^{*}(u, y) q>
$$

and is given by

$$
\begin{aligned}
& \Lambda^{*}(u, y) q(x) \\
& =\int_{\Re^{\mathrm{n}}} \frac{\left.\exp \|-\frac{1}{2 \varepsilon}\left(x-f_{d}(z, u)\right)^{T}\left(g_{d} W g_{d}^{T}\right)^{-1}\left(x-f_{d}(z, u)\right)\right\}}{(2 \pi)^{p / 2}|\varepsilon W|^{1 / 2}} \\
& \quad \times q(z) \exp \left(\frac{\theta}{\varepsilon} M(u)\right) \psi(z, y) d z .
\end{aligned}
$$

Proof: It follows from (26) that

$<q\left(x, k h^{+}\right), \phi>$

$=\tilde{E}\left[\phi\left(f_{d}(x, u, k h)+g_{d}(x, k h)\right) \psi\left(x(k h), y_{k+1}\right)\right.$

$\left.\times \exp \left(\frac{\theta}{\varepsilon} M\left(u_{k}\right)\right) \eta_{k} \exp \left(\frac{\theta}{\varepsilon} \sum_{l=0}^{k} M\left(u_{l}\right)\right) \mid \mathcal{Y}^{k+1}\right]$

$=\tilde{E}\left[\int_{\Re^{n}} \frac{\exp \left(-\frac{1}{2 \varepsilon} w^{T} W^{-1} w\right)}{(2 \pi)^{p / 2}|\varepsilon W|^{1 / 2}} \phi\left(f_{d}(x, u, k h)\right.\right.$

$\left.+g_{d}(x, k h)\right) \psi\left(x(k h), y_{k+1}\right) \exp \left(\frac{\theta}{\varepsilon} M\left(u_{k}\right)\right) \eta_{k}$

$\left.\times \exp \left(\frac{\theta}{\varepsilon} \sum_{l=0}^{k} M\left(u_{l}\right)\right) d w \mid \mathcal{Y}^{k+1}\right]$

$=<q(x, k h), \quad \int_{\Re^{n}} \frac{\exp \left(-\frac{1}{2 \epsilon} w^{T} W^{-1} w\right)}{(2 \pi)^{p / 2}|\varepsilon W|^{1 / 2}} \phi\left(f_{d}(x, u, k h)\right.$

$\left.+g_{d}(x, k h)\right) \psi\left(x(k h), y_{k+1}\right) \exp \left(\frac{\theta}{\varepsilon} M\left(u_{k}\right)\right) d w>$

$=<q(x, k h), \Lambda\left(u_{k}, y_{k+1}\right) \phi>$

$=<\Lambda^{*}\left(u_{k}, y_{k+1}\right) q(x, k h), \phi>$.

Q.E.D.

Remark 2.2 There exists a backward adjoint process $p(x, k h)$ such that

$$
\begin{aligned}
<\Lambda^{*} q, p> & =<q, \Lambda p>, t \neq k h \\
<q\left(x, k h^{+}\right), & p\left(x, k h^{+}\right)> \\
& =<q(x, k h), p(x, k h)>, t=k h .
\end{aligned}
$$

for all $k$. Actually, the process $p(x, k h)$ at time $k h$ satisfies the recursive equation adjoint to (28), namely,

$$
p(x, k h)=\Lambda\left(u_{k}, y_{k+1}\right) p\left(x, k h^{+}\right)
$$

Using the information state $q(x, t)$, we obtain the representation of the cost criterion

$$
J(u)=\tilde{E}\left[\int_{\Re^{n}} \exp \left(\frac{\theta}{\varepsilon} \Phi\left(x, t_{f}\right)\right) q\left(x, t_{f}\right) d x\right] .
$$

Due to the representation (34), the problem is now to minimize the cost criterion (34) subject to the dynamics (21), (22) and (26).

\subsection{Dynamic Programming}

Since the information state defined in Section 2.2 can be considered as the state of the system, we can easily write down Dynamic Programming equation. We define the optimal value function by

$$
\begin{aligned}
& V(q, t)= \\
& \inf _{u} \tilde{E}\left[\int_{\Re^{n}} \exp \left(\frac{\theta}{\varepsilon} \Phi\left(x, t_{f}\right)\right) q\left(x, t_{f}\right) d x \mid q(x, t)=q\right] .
\end{aligned}
$$

Suppose that $V(q, t)$ is twice differentiable in $q$ and differentiable in $t$. Then, $V(q, t)$ satisfies the HamiltonJacobi-Bellman(HJB) equations

$$
\begin{aligned}
-\frac{\partial V}{\partial t}= & D_{q} V\left\{-D_{x} q(x, t) f(x, t)\right. \\
& -q(x, t) \operatorname{tr} D_{x} f(x, t)+\frac{\theta}{\varepsilon} q(x, t) \\
& \left.+\frac{\varepsilon}{2} \operatorname{trg}(x) W g^{T}(x) D_{x}^{2} q(x, t)\right\}, \\
V(q, k h) & =\inf _{u} \tilde{E}\left[\exp \left(\frac{\theta}{\varepsilon} u_{k}^{T} R_{k} u_{k}\right) V\left(q, k h^{+}\right)\right], \\
V\left(q, t_{f}\right) & =\int_{\Re^{n}} \exp \left(\frac{\theta}{\varepsilon} \Phi\left(x, t_{f}\right)\right) q\left(x, t_{f}\right) d x .
\end{aligned}
$$

The optimal control $u^{*}$ is the feedback control which minimizes the right-hand-side of (37).

\section{$3 \quad$ Linear Exponential Quadratic Gaussian Control Problem}

\subsection{Problem Formulation}

We consider the linear exponential quadratic Gaussian control problem. The system (1)-(4) is specialized to the following linear system.

$$
\begin{aligned}
& d x=A(t) x(t) d t+\sqrt{\varepsilon} D(t) d w, t \neq k h, \\
& x\left(k h^{+}\right)=A_{d k} x(k h)+B_{d k} u_{k}+\sqrt{\varepsilon} D_{d k} w_{k}, \\
& x(0)=x_{0}+\sqrt{\varepsilon} \hat{x}_{0} . \\
& y_{k+1}=H_{k} x(k h)+\sqrt{\varepsilon} v_{k},
\end{aligned}
$$

The cost criterion is also specialized to the quadratic quantity.

$$
\begin{aligned}
J(u) & =E \exp \left[\frac { \theta } { 2 \varepsilon } \left[x^{T}\left(t_{f}\right) Q_{f} x\left(t_{f}\right)\right.\right. \\
& \left.\left.+\int_{0}^{t_{f}} x^{T}(t) Q(t) x(t) d t+\sum_{k=0}^{N-1} u_{k}^{T} R_{k} u_{k}\right]\right] .
\end{aligned}
$$

\subsection{Information State}

For the linear case, the information state $q(x, t)$, which satisfies (21) and (22), can be explicitly expressed.

Theorem 3. 1 The solution to the partial differential equation (21) with (22) is

$$
q(x, t)=\frac{\nu_{t} \exp \left\{-\frac{1}{2}(x-\hat{x})^{T} P^{-1}(x-\hat{x})\right\}}{(2 \pi)^{n / 2}|P|^{1 / 2}}
$$

where $\hat{x}$ is given by

$d \hat{x}=\left(A+\frac{\theta}{\varepsilon} P Q\right) \hat{x} d t$

with $\hat{x}(0)=\hat{x}_{0}$, and $P$ satisfies the Riccati equation 
$\dot{P}=A P+P A^{T}+\varepsilon D W D^{T}+\frac{\theta}{\varepsilon} P Q P$,

$P(0)=\varepsilon P_{0}$,

and $\nu_{t}$ is given by

$\nu_{t}=\exp \left[\frac{\theta}{2 \varepsilon} \int_{0}^{t}\left(\hat{x}^{T} Q \hat{x}+\operatorname{tr} P Q\right) d \tau\right]$.

Proof: Differentiating $q^{\theta, \varepsilon}(x, t)$ defined by (44), (45), (46) and (47), and substituting in (21) verify the result. Q.E.D.

We can also express $p^{\theta, \varepsilon}(x, t)$ in terms of $x^{\varepsilon}$ due to the linearity of the dynamical system (39) and (41).

Theorem 3.2 The process $p^{\theta, \varepsilon}(x, t)$ can be uritten as

$$
p(x, t)=\exp \left(\frac{\theta}{2 \varepsilon} x^{T} K x+\frac{\theta}{2} \int_{t}^{t_{f}} \operatorname{tr} D W D^{T} K^{\theta} d \tau\right)
$$

where $K$ is given by

$$
\begin{aligned}
& -\dot{K}=A^{T} K+K A+Q+\theta K D W D^{T} K, \\
& K\left(t_{f}\right)=Q_{f} .
\end{aligned}
$$

Proof: Differentiating $p(x, t)$ defined by (48) and (49), and substituting in (24) verify the result. Q.E.D.

At time $k h$, we can also solve the recursive equations (28) and (33) for the information state $q(x, k h)$ and its adjoint $p(x, k h)$.

Theorem 3. 3 The process $q(x, k h)$ at time $k h$ is given by

$$
\begin{aligned}
& q(x, k h) \\
& =\frac{\nu(k h) \exp \left\{-\frac{1}{2}(x(k h)-\hat{x}(k h))^{T} P^{-1}(k h)(x(k h)-\hat{x}(k h))\right\}}{(2 \pi)^{n / 2}|P(k h)|^{1 / 2}}
\end{aligned}
$$

where $\hat{x}(k h)$ is given by

$$
\begin{aligned}
& \hat{x}\left(k h^{+}\right)=A_{d k} \hat{x}(k h)+B_{d k} u_{k} \\
& \quad+\frac{1}{\varepsilon} A_{d k}\left(P^{-1}(k h)+\frac{1}{\varepsilon} H_{k}^{T} V_{k}^{-1} H_{k}\right)^{-1} H_{k}^{T} V_{k}^{-1} \\
& \quad \times\left(y_{k+1}-H_{k} \hat{x}(k h)\right)
\end{aligned}
$$

with $\hat{x}(0)=\hat{x}_{0}$, and $P(k h)$ satisfies the Riccati equation

$$
\begin{gathered}
P\left(k h^{+}\right)=\varepsilon D_{d k} W_{k} D_{d k}^{T}+A_{d k}\left(P^{-1}(k h)\right. \\
\left.\quad+\frac{1}{\varepsilon} H_{k}^{T} V_{k}^{-1} H_{k}\right)^{-1} A_{d k}^{T}, P(0)=\varepsilon P_{0},
\end{gathered}
$$

and $\nu(k h)$ is given by

$$
\begin{gathered}
\nu(k h)=\beta_{k}\left(\Pi_{l=1}^{k} \psi\left(\hat{x}((l-1) h), y_{l}\right)\right) \\
\quad \times \exp \left(\frac{\theta}{2 \varepsilon} \sum_{l=0}^{k-1} u_{l}^{T} R_{l} u_{l}\right),
\end{gathered}
$$

$\beta_{k+1}=\beta_{k}^{\varepsilon}\left|\varepsilon W_{k} S_{k}\right|^{-1 / 2} \Pi_{l=1}^{k+1} \exp \left[\frac{1}{2 \varepsilon^{2}}\left(y-H_{l} \hat{x}^{\varepsilon}\right)^{T}\right.$

$\times V_{l}^{-1} H_{l}\left(\left(P^{\varepsilon}\right)^{-1}(l h)+\frac{1}{\varepsilon} H_{l}^{-1} V_{l}^{-1} H_{l}\right)^{-1} H_{l}^{T} V_{l}^{-1}$ $\left.\times\left(y-H_{l} \hat{x}\right)\right]$,

$$
\begin{aligned}
S_{k}= & \frac{1}{\varepsilon} A_{d k}^{T}\left(D_{d k} W_{k} D_{d k}^{T}\right)^{-1} A_{d k}+P^{-1}(k h) \\
& +\frac{1}{\varepsilon} H_{k}^{T} V_{k}^{-1} H_{k} .
\end{aligned}
$$

Proof: The form of (50) obviously holds at time $t=0$. Suppose it holds at time $t=k h$. Then we calculate the right-hand-side of (28) as

$$
\begin{aligned}
& \Lambda^{*}(u, y) q(x) \\
& =\frac{\nu(k h) \exp \left(\frac{\theta}{2 \varepsilon} u_{k}^{T} R_{k} u_{k}\right)\left|S_{k}\right|^{-1 / 2}}{(2 \pi)^{p / 2 \mid}\left|\epsilon W_{k} P(k h)\right|{ }^{1 / 2}} \int_{\Re^{n}} \frac{1}{(2 \pi)^{n / 2}\left|S_{k}\right|^{-1 / 2}} \\
& \quad \times \exp \left\{-\frac{1}{2 \varepsilon}\left(z-\bar{z}_{k}\right)^{T} S_{k}\left(z-\bar{z}_{k}\right)\right\} d z \\
& \quad \times \exp \left\{-\frac{1}{2 \varepsilon}\left(x-B_{d k} u\right)^{T}\left(D_{d k} W_{k} D_{d k}^{T}\right)^{-1}\right. \\
& \left.\quad \times\left(x-B_{d k} u\right)-\frac{1}{2} \hat{x}^{T} P^{-1}(k h) \hat{x}+\frac{1}{2} \bar{z}_{k}^{T} S_{k} \bar{z}_{k}\right\}
\end{aligned}
$$

where

$$
\begin{aligned}
\bar{z}_{k}= & S_{k}^{-1}\left[\frac{1}{\varepsilon} A_{d k}^{T}\left(D_{d k} W_{k} D_{d k}^{T}\right)^{-1}\left(x-B_{d k} u\right)\right. \\
& \left.+P^{-1}(k h) \hat{x}+\frac{1}{\varepsilon} H_{k}^{T} V_{k}^{-1} y\right] .
\end{aligned}
$$

The integral in the above equation (56) is equal to one. Hence we have

$$
\begin{aligned}
& \Lambda^{*}(u, y) q(x)=\frac{\nu(k h) \exp \left(\frac{\theta}{2 \varepsilon} u_{k}^{T} R_{k} u_{k}\right)\left|S_{k}\right|^{-1 / 2}}{(2 \pi)^{p / 2}\left|\varepsilon W_{k} P(k h)\right|^{1 / 2}} \\
& \quad \times \exp \left[-\frac{1}{2}\left(x-\hat{x}\left(k h^{+}\right)\right)^{T} P^{-1}\left(k h^{+}\right)\left(x-\hat{x}\left(k h^{+}\right)\right)\right. \\
& \quad+\frac{1}{\varepsilon}\left(\hat{x}^{T} H_{k}^{T} V_{k}^{-1} y-\frac{1}{2} \hat{x}^{T} H_{k}^{T} V_{k}^{-1} H_{k} \hat{x}\right) \\
& \quad+\frac{1}{2 \varepsilon^{2}}\left(y-H_{k} \hat{x}\right)^{T}\left[V _ { k } ^ { - 1 } H _ { k } \left(P^{-1}(k h)\right.\right. \\
& \left.\left.\quad+\frac{1}{\varepsilon} H_{k}^{-1} V_{k}^{-1} H_{k}\right)^{-1} H_{k}^{T} V_{k}^{-1}\right]\left(y-H_{k} \hat{x}\right)
\end{aligned}
$$

where

$$
\begin{aligned}
& \hat{x}\left(k h^{+}\right)=\frac{1}{\varepsilon} P\left(k h^{+}\right)\left(D_{d k} W_{k} D_{d k}^{T}\right)^{-1}[\{I \\
& \left.\quad-\frac{1}{\varepsilon} A_{d k} S_{k}^{-1} A_{d k}^{T}\left(D_{d k} W_{k} D_{d k}^{T}\right)^{-1}\right\} B_{d k} u \\
& \left.\quad+\frac{1}{\varepsilon} A_{d k} S_{k}^{-1}\left(P^{-1}(k h) \hat{x}+\frac{1}{\varepsilon} H_{k}^{T} V_{k}^{-1} y\right)\right], \\
& P\left(k h^{+}\right)=\left[\frac{1}{\varepsilon}\left(D_{d k} W_{k} D_{d k}^{T}\right)^{-1}\right. \\
& \quad-\frac{1}{\epsilon^{2}}\left(D_{d k} W_{k} D_{d k}^{T}\right)^{-1} \\
& \left.\quad \times A_{d k} S_{k}^{-1} A_{d k}^{T}\left(D_{d k} W_{k} D_{d k}^{T}\right)^{-1}\right]^{-1}
\end{aligned}
$$

We apply the matrix inversion lemma to $P\left(k h^{+}\right)$and then have (52). (51) readily follows. This is the end of the proof. Q.E.D.

Theorem 3.4 The process $p(x, k h)$ at time $k h$ is given by

$$
\begin{aligned}
p(x, k h) & =\beta_{k}\left(\Pi_{l=k+1}^{N-1} \psi\left(x((l-1) h), y_{l}\right)\right) \\
& \times \exp \left[\frac { \theta } { 2 \varepsilon } \left\{x^{T}(k h) K(k h) x(k h)\right.\right. \\
& \left.\left.+\sum_{l=k}^{N-1}\left(u_{l}-u_{l}^{*}\right)^{T} R_{l}\left(u_{l}-u_{l}^{*}\right)\right\}\right]
\end{aligned}
$$

where $\beta_{k}, K(k h)$ and $u_{k}^{*}$ satisfy

$$
\begin{array}{r}
\beta(k h)=\beta\left(k h^{+}\right) \sqrt{\frac{\left|W_{k}^{-1}-\theta D_{d k}^{T} K\left(k h^{+}\right) D_{d k}\right|^{-1}}{\left|W_{k}\right|}}, \\
K(k h)=A_{d k}^{T}\left(K^{-1}\left(k h^{+}\right)+B_{d k} R_{k}^{-1} B_{d k}^{T}\right. \\
\left.-\theta D_{d k} W_{k} D_{d k}^{T}\right)^{-1} A_{d k}, \\
u_{k}^{*}=-R_{k}^{-1} B_{d k}^{T}\left(K^{-1}\left(k h^{+}\right)+B_{d k} R_{k}^{-1} B_{d k}^{T}\right. \\
\left.-\theta D_{d k} W_{k} D_{d k}^{T}\right)^{-1} A_{d k} x(k h) .
\end{array}
$$

Before we prove the above theorem, we give the following lemma.

Lemma 3.1 Let $K(k h)$ satisfy the discrete-time Riccati equations (63). If $W_{k}^{-1}-\theta D_{d k}^{T} K(k h) D_{d k}>0$, then

$$
\begin{aligned}
& \int_{-\infty}^{\infty} \frac{1}{\sqrt{(2 \pi)^{p}\left|W_{k}\right|}} \exp \left[-\frac{1}{2} w_{k}^{T} W_{k}^{-1} w_{k}\right] \\
& \quad \times \exp \left[\frac{\theta}{2 \varepsilon} x\left(k h^{+}\right)^{T} K\left(k h^{+}\right) x\left(k h^{+}\right)\right] d w_{k} \\
& =\sqrt{\frac{\left|W_{k}^{-1}-\theta D_{d k}^{T} K\left(k h^{+}\right) D_{d k}\right|^{-1}}{\left|W_{k}\right|}} \exp \left[\frac { \theta } { 2 \varepsilon } \left(A_{d k} x(k h)\right.\right. \\
& \left.\left.\quad+B_{d k} u_{k}\right)^{T} \bar{K}_{k}\left(A_{d k} x(k h)+B_{d k} u_{k}\right)\right]
\end{aligned}
$$

where

$$
\begin{aligned}
\bar{K}_{k}=\left(K^{-1}\left(k h^{+}\right)+\right. & B_{d k} R_{k}^{-1} B_{d k}^{T} \\
& \left.-\theta D_{d k} W_{k} D_{d k}^{T}\right)^{-1},
\end{aligned}
$$


Proof: See [3].

Proof of Theorem 3.4: Substitution of (61) into (33) and using the lemma yield

$$
\begin{aligned}
& \Lambda\left(u_{k}, y_{k+1}\right) p\left(x, k h^{+}\right) \\
& =\beta\left(k h^{+}\right) \sqrt{\frac{\left|W_{k}^{-1}-\theta D_{d k}^{T} K\left(k h^{+}\right) D_{d k}\right|^{-1}}{\left|W_{k}\right|}} \\
& \quad \times \exp \left[\frac { \theta } { 2 \varepsilon } ( A _ { d k } x ( k h ) + B _ { d k } u _ { k } ) ^ { T } \overline { K } _ { k } \left(A_{d k} x(k h)\right.\right. \\
& \left.\left.\quad+B_{d k} u_{k}\right)\right]\left(\Pi_{l=k+1}^{N-1} \psi\left(x((l-1) h), y_{l}\right)\right) \\
& \left.\quad \times \exp \left[\frac{\theta}{2 \varepsilon} \sum_{l=k+1}^{N-1}\left(u_{l}-u_{l}^{*}\right)^{T} R_{l}\left(u_{l}-u_{l}^{*}\right)\right\}\right] \\
& \quad \times \exp \left(\frac{\theta}{2 \varepsilon} u_{k}^{T} R_{k} u_{k}\right) \\
& =\beta(k h)\left(\Pi_{l=k+1}^{N-1} \psi\left(x((l-1) h), y_{l}\right)\right) \\
& \quad \times \exp \left[\frac { \theta } { 2 \varepsilon } \left\{x^{T}(k h) K(k h) x(k h)\right.\right. \\
& \left.\left.\quad+\sum_{l=k}^{N-1}\left(u_{l}-u_{l}^{*}\right)^{T} R_{l}\left(u_{l}-u_{l}^{*}\right)\right\}\right] .
\end{aligned}
$$

Thus we have the desired result. Q.E.D.

\subsection{Cost Criterion}

Now we can rewrite the cost criterion.

Theorem 3.5 The cost criterion can be written as

$$
\begin{aligned}
& J(u)=E\left[\operatorname { e x p } \left\{\frac { \theta } { 2 \varepsilon } \left(\hat{\phi}\left(x\left(t_{f}\right), P\left(t_{f}\right)\right)\right.\right.\right. \\
& \left.\left.\left.\quad+\int_{0}^{t_{f}}\left(\hat{x}^{T} Q \hat{x}+\operatorname{tr} P Q\right) d t+\sum_{k=0}^{N-1} u_{k}^{T} R_{k} u_{k}\right)\right\}\right]
\end{aligned}
$$

where

$$
\begin{aligned}
& \hat{\phi}(x, P)=\frac{2 \varepsilon}{\theta} \log \left|I-\frac{\theta}{\varepsilon} P Q\right|^{-1 / 2}+\hat{x}^{T} \hat{Q}_{f} \hat{x}, \\
& \hat{Q}_{f}=\frac{1}{2}\left[\left(I-\frac{\theta}{\varepsilon} Q_{f} P\right)^{-1} Q_{f}+Q_{f}\left(I-P \frac{\theta}{\varepsilon} Q_{f}\right)^{-1}\right] .
\end{aligned}
$$

Proof: Using Theorem 3.1 and Theorem 3.2, the cost criterion can be written as

$$
\begin{aligned}
& J(u)=\tilde{E}\left[\int_{\Re^{n}} p\left(x, t_{f}\right) q\left(x, t_{f}\right) d x\right] \\
& =\tilde{E}\left[\int_{\Re^{n}} \exp \left(\frac{\theta}{2 \varepsilon} x^{T} Q_{f} x\right)\right. \\
& \left.\quad \times \frac{\exp \left[\frac{\theta}{2 \varepsilon}\left\{\int_{0}^{t_{f}}\left(\hat{x}^{T} Q \hat{x}+\operatorname{tr} P Q\right) d t+\sum_{k=0}^{N-1} u_{k}^{T} R_{k} u_{k}\right\}\right.}{(2 \pi)^{n / 2}|P|^{1 / 2}} d x\right] \\
& \quad \times \exp \left\{-\frac{1}{2}(x-\hat{x})^{T} P^{-1}(x-\hat{x})\right\} \\
& =E\left[\operatorname { e x p } \left\{\frac{\theta}{2 \varepsilon} \hat{\phi}(\hat{x}, P)+\frac{\theta}{2 \varepsilon}\left\{\int_{0}^{t_{f}}\left(\hat{x}^{T} Q \hat{x}+\operatorname{tr} P Q\right) d t\right.\right.\right. \\
& \left.\left.\quad+\sum_{k=0}^{N-1} u_{k}^{T} R_{k} u_{k}\right\}\right] . \\
& \text { Q.E.D. }
\end{aligned}
$$

\subsection{Solution to LEQG Control Problem}

Thus our original problem is restated as follows; minimize the cost criterion $J(u)$ defined by (68) and (69), subject to dynamics

$$
\begin{gathered}
d \hat{x}=\left(A+\frac{\theta}{\varepsilon} P Q\right) \hat{x} d t, \hat{x}(0)=\hat{x}_{0}, t \neq k h, \\
\hat{x}\left(k h^{+}\right)=A_{d k} \hat{x}(k h)+B_{d k} u_{k}+\frac{1}{\varepsilon} A_{d k}\left(P^{-1}(k h)\right. \\
\left.\quad+\frac{1}{\varepsilon} H_{k}^{T} V_{k}^{-1} H_{k}\right)^{-1} H_{k}^{T} V_{k}^{-1}\left(y_{k+1}-H_{k} \hat{x}(k h)\right), \\
t=k h, \\
\dot{P}=A P+P A^{T}+\varepsilon D W D^{T}+\frac{\theta}{\varepsilon} P Q P, t \neq k h, \\
P(0)=\varepsilon P_{0}, \\
P\left(k h^{+}\right)=\varepsilon D_{d k} W_{k} D_{d k}^{T}+A_{d k}\left(P^{-1}(k h)\right. \\
\left.\quad+\frac{1}{\varepsilon} H_{k}^{T} V_{k}^{-1} H_{k}\right)^{-1} A_{d k}^{T}, t=k h .
\end{gathered}
$$

Suppose that $\bar{V}(\hat{x}, P, t)$ is differentiable in $t, \hat{x}$ and $P$. The Hamilton-Jacobi-Bellman equation for this altered problem can be written as

$$
\begin{aligned}
& -\frac{\partial \bar{V}}{\partial t}=D_{\hat{x}} \bar{V}\left(A+\frac{\theta}{\varepsilon} P Q\right) \hat{x} \\
& \quad+\frac{\theta}{2 \varepsilon} \bar{V}\left(\hat{x}^{T} Q \hat{x}+\operatorname{tr} P Q\right), t \neq k h, \\
& \bar{V}(\hat{x}, P, k h)=\inf _{u} E\left[\exp \left(\frac{\theta}{2 \varepsilon} u_{k}^{T} R_{k} u_{k}\right) \bar{V}\left(k h^{+}\right)\right], \\
& t=k h . \\
& \bar{V}\left(\hat{x}, P, t_{f}\right)=\frac{1}{\left|I-\frac{\theta}{\varepsilon} P Q\right|} \exp \left(\frac{\theta}{2 \varepsilon} \hat{x}^{T}\left(t_{f}\right) \hat{Q}_{f} \hat{x}\left(t_{f}\right)\right),
\end{aligned}
$$

Theorem 3.6 The optimal control for the risksensitive control problem is given by

$$
\begin{aligned}
u_{k}^{*}= & -R_{k}^{-1} B_{d k}^{T}\left(\Pi^{-1}\left(k h^{+}\right)+B_{d k} R_{k}^{-1} B_{d k}^{T}\right. \\
& \left.-\theta D_{d k} W_{k}^{-1} D_{d k}^{T}\right)^{-1} A_{d k} \hat{x}(k h)
\end{aligned}
$$

where $\Pi(k h)$ satisfies

$$
\begin{aligned}
& -\dot{\Pi}=\left(A+\frac{\theta}{\varepsilon} P Q\right)^{T} \Pi+\Pi\left(A+\frac{\theta}{\varepsilon} P Q\right)+Q, \\
& \Pi\left(t_{f}\right)=\hat{Q}_{f}, t \neq k h, \\
& \Pi(k h)=A_{d k}^{T}\left(\Pi\left(k h^{+}\right)^{-1}+B_{d k} R_{k}^{-1} B_{d k}^{T}\right. \\
& \left.\quad-\theta D_{d k} W_{k}^{-1} D_{d k}^{T}\right)^{-1} A_{d k}, t=k h .
\end{aligned}
$$

Proof: (75) can be solved by assuming the form of a solution

$$
\bar{V}(\hat{x}, P, t)=\beta(t) \exp \left(\frac{\theta}{2 \varepsilon} \hat{x}^{T}(t) \Pi(t) \hat{x}(t)+\alpha(t)\right) .
$$

Substitution of (81) into (75) verifies

$$
\begin{aligned}
\bar{V}(\hat{x}, P, t)= & \frac{1}{\left|I-\frac{\theta}{\varepsilon} P Q\right|} \exp \left(\frac{\theta}{2 \epsilon} \hat{x}^{T}(t) \Pi(t) \hat{x}(t)\right. \\
& \left.+\frac{1}{2} \int_{t}^{t_{f}} \operatorname{tr} P Q d \tau\right)
\end{aligned}
$$

where $\Pi(t)$ satisfies (79). Substitution of (81) into (76) and making some manipulations give

$$
\begin{aligned}
& \bar{V}(\hat{x}, P, k h)=\inf _{u}\left[\beta\left(k h^{+}\right)\right. \\
& \times \sqrt{\frac{\left|W_{k}^{-1}-\theta D_{d k}^{T} K\left(k h^{+}\right) D_{d k}\right|^{-1}}{\left|W_{k}\right|}} \exp \left[\frac { \theta } { 2 \varepsilon } \left(u_{k}^{T} R_{k} u_{k}\right.\right. \\
& \left.+\left(A_{d k} x(k h)+B_{d k} u_{k}\right)^{T} \bar{K}_{k}\left(A_{d k} x(k h)+B_{d k} u_{k}\right)\right] .
\end{aligned}
$$

Minimization of the right-hand-side of (83) with respect to $u_{k}$ provides the optimal control $u_{k}^{*}$. Moreover, substitution of $u_{k}^{*}$ back into (83) and comparison of both sides of (83) gives (80). Q.E.D.

\section{Asymptotic Analysis of Risk- sensitive Controller}

We investigate asymptotic limits of the risk-sensitive controller given by (78) when $\varepsilon$ and $\theta$ tend to zero.

\subsection{Small Noise Limit}

We consider the small noise limit when $\varepsilon$ goes to zero. First consider the Riccati equations (46) and (52). Taking $\varepsilon \rightarrow 0$, we have

$$
\frac{P}{\varepsilon} \rightarrow P \text {. }
$$

Thus we have the following results.

Theorem 4.1 The risk-sensitive optimal control in the small noise limit is given by 


$$
\begin{aligned}
& u_{k}^{*}=-R_{k}^{-1} B_{d k}^{T}\left(\Pi^{-1}\left(k h^{+}\right)+B_{d k} R_{k}^{-1} B_{d k}^{T} \cdot\right. \\
&\left.-\theta D_{d k} W_{k}^{-1} D_{d k}^{T}\right)^{-1} A_{d k} \hat{x}(k h), \\
& d \hat{x}=\left(A+\theta P^{\theta} Q\right) \hat{x} d t, t \neq k h, \\
& \hat{x}\left(k h^{+}\right)=A_{d k} \hat{x}(k h)+B_{d k} u_{k} \\
&+A_{d k}\left(P^{-1}(k h)+H_{k}^{T} V_{k}^{-1} H_{k}\right)^{-1} H_{k}^{T} V_{k}^{-1} \\
& \quad \times\left(y_{k+1}-H_{k} \hat{x}(k h)\right)
\end{aligned}
$$

with $\hat{x}(0)=\hat{x}_{0}$. Here $P$ and $\Pi$ satisfy

$$
\begin{aligned}
& \dot{P}=A P+P A^{T}+D W D^{T}+\theta P Q P, \\
& P(0)=P_{0}, t \neq k h \text {, } \\
& P\left(k h^{+}\right)=D_{d k} W_{k} D_{d k}^{T}+A_{d k}\left(P^{-1}(k h)\right. \\
& \left.+H_{k}^{T} V_{k}^{-1} H_{k}\right)^{-1} A_{d k}^{T}, t=k h \text {, } \\
& -\dot{\Pi}=(A+\theta P Q)^{T} \Pi+\Pi(A+\theta P Q)+Q, \\
& \Pi\left(t_{f}\right)=\hat{Q}_{f}, t \neq k h \text {, } \\
& \Pi(k h)=A_{d k}^{T}\left(\Pi\left(k h^{+}\right)^{-1}+B_{d k} R_{k}^{-1} B_{d k}^{T}\right. \\
& \left.-\theta D_{d k} W_{k}^{-1} D_{d k}^{T}\right)^{-1} A_{d k}, t=k h \text {. }
\end{aligned}
$$

Remark 4. 1 The control $u_{k}^{*}$ in (85) is an $H_{\infty}$ controller for sampled-data systems.

\subsection{Small Risk Limit}

We next consider the small risk limit when $\theta$ goes to zero. Consider the Riccati equations (46) and (52) again. Taking $\theta \rightarrow 0$, we have the following results.

Theorem 4.2 The risk-sensitive optimal control in the small risk limit is given by

$$
\begin{aligned}
u_{k}^{*}= & -R_{k}^{-1} B_{d k}^{T}\left(\Pi^{-1}\left(k h^{+}\right)\right. \\
& \left.+B_{d k} R_{k}^{-1} B_{d k}^{T}\right)^{-1} A_{d k} \hat{x}(k h), \\
d \hat{x} \doteq & A \hat{x} d t, t \neq k h, \\
\hat{x}( & \left.k h^{+}\right)=A_{d k} \hat{x}(k h)+B_{d k} u_{k} \\
& +\frac{1}{\varepsilon} A_{d k}\left(P^{-1}(k h)+\frac{1}{\varepsilon} H_{k}^{T} V_{k}^{-1} H_{k}\right)^{-1} H_{k}^{T} V_{k}^{-1} \\
& \times\left(y_{k+1}-H_{k} \hat{x}(k h)\right)
\end{aligned}
$$

with $\hat{x}(0)=\hat{x}_{0}$. Here $P$ and $\Pi$ satisfy

$$
\begin{aligned}
& \dot{P}=A P+P A^{T}+\varepsilon D W D^{T}, \\
& P(0)=P_{0}, t \neq k h \text {, } \\
& P\left(k h^{+}\right)=\varepsilon D_{d k} W_{k} D_{d k}^{T}+A_{d k}\left(P^{-1}(k h)\right. \\
& \left.+H_{k}^{T} V_{k}^{-1} H_{k}\right)^{-1} A_{d k}^{T}, \quad t=k h, \\
& -\dot{\Pi}=A^{T} \Pi+\Pi A+Q, \Pi\left(t_{f}\right)=\hat{Q}_{f}, t \neq k h, \\
& \Pi(k h)=A_{d k}^{T}\left(\Pi\left(k h^{+}\right)^{-1}+B_{d k} R_{k}^{-1} B_{d k}^{T}\right)^{-1} A_{d k}, \\
& t=k h \text {. }
\end{aligned}
$$

Remark 4.2 The control $u_{k}^{*}$ in (92) is a stochastic optimal controller for sampled-data systems.

\section{Application to Sampled-data Systems}

We have mainly considered the problem for jump systems. However, we can easily extend the results to sampled-data systems by defining the extended state. Define

$$
\dot{\bar{x}}=0, k h<t \leq(k+1) h, \bar{x}=u_{k} \text {. }
$$

Then the system (39)-(42) can be written with $x_{\boldsymbol{e}}=$ $\left[x^{T} \bar{x}^{T}\right]$ and

$$
\begin{aligned}
& A=\left[\begin{array}{cc}
A & B_{d k} \\
0 & 0
\end{array}\right], A_{d k}=\left[\begin{array}{cc}
A_{d k} & 0 \\
0 & 0
\end{array}\right], \\
& B_{d k}=\left[\begin{array}{l}
0 \\
I
\end{array}\right], D=\left[\begin{array}{c}
D \\
0
\end{array}\right], \\
& D=\left[\begin{array}{c}
D \\
0
\end{array}\right], H_{k}=\left[\begin{array}{ll}
H_{k} & 0
\end{array}\right] .
\end{aligned}
$$

The problem can be solved by using the result obtained for jump systems.

\section{Conclusion}

We have considered the Risk-sensitive control problem for nonlinear sampled-data systems. We employed the information state to analyze the problem, and explicitly obtained the LEQG optimal controller. We have also investigated the various limits of the controller.

\section{References}

[1] A. Bensoussan, Stochastic Control of Partially Observable Systems, Cambridge University Press, 1992.

[2] A. Bensoussan and J.H. van Shuppen, Optimai Control of Partially Observable Stochastic Systems with an Exponential-of-integral Performance Index, SIAM Journal of Control and Optimization, vol.23, no.4, 599-613, 1985.

[3] D.H. Jacobson, Optimal Stochastic Linear Systems with Exponential Criteria and Their Relation to Deterministic Differential Games, IEEE Transaction on Automatic Control, vol.18, no.2, 124-131, 1973.

[4] P.R. Kumar and P. Varaiya, Stochastic Systems Estimation, Identification, and Adaptive Control, Prentice Hall, 1986.

[5] J.L. Speyer, An Adaptive Terminal Guidance Scheme Based on an Exponential Cost Criterion with Applications to Homing Missile Guidance, IEEE Transaction on Automatic Control, vol.21, no.9, 1021-1032, 1976.

[6] P. Whittle, Risk-sensitive Linear /Quadratic /Gaussian Control, Adv. Appli. Prob., vol.13, 764777, 1981. 